\title{
Le cycle de l'eau à grande échelle : impact de changements climatiques globaux
}

\author{
par R. Sadourny \\ Laboratoire de Météorologie Dynamique du CNRS \\ École Normale Supérieure, Paris
}

\section{INTRODUCTION}

Le cycle de l'eau joue un rôle central dans la dynamique du climat terrestre. La vapeur d'eau dans l'atmosphère est le plus abondant et le plus efficace des gaz à effet de serre, loin devant le dioxyde de carbone. Elle joue donc un rôle de premier plan dans le bilan radiatif global de la planète : le flux vers le bas dans l'infrarouge dû à l'effet de serre de la vapeur d'eau (qui correspond à un réchauffement de la surface) est en moyenne de l'ordre de $100 \mathrm{~W} \mathrm{~m}^{-2}$, valeur à comparer aux $240 \mathrm{~W} \mathrm{~m}^{-2}$ du flux solaire net absorbé ; à cet effet de serre de la vapeur d'eau, il faut ajouter l'effet de serre des nuages, qui correspond à un flux vers le bas dans l'infrarouge de $31 \mathrm{~W} \mathrm{~m}^{-2}$ environ. En plus de leur rôle dans l'effet de serre, les nuages réfléchissent l'énergie solaire ; cet effet entraîne un déficit d'ensoleillement des basses couches, ou en d'autres termes un refroidissement de la surface, qui correspond à un flux vers le haut de $47 \mathrm{~W} \mathrm{~m}^{-2}$. Les effets énergétiques du cycle de l'eau ne se limitent d'ailleurs pas aux aspects radiatifs, car la vapeur d'eau transporte aussi de l'énergie sous forme de chaleur latente. Cette énergie latente, alimentée par l'évaporation à la surface, est transportée par les mouvements de l'air et se libère lors de la condensation sous forme de gouttelettes d'eau ou de cristaux de neige ou de glace. Le flux d'énergie correspondant, donné par l'évaporation moyenne globale, se situe aux environs de $82 \mathrm{~W}$ $\mathrm{m}^{-2}$. On voit que l'ensemble de ces processus mettent en jeu des flux d'énergie voisins du flux d'énergie solaire.

Cette prééminence du cycle de l'eau dans l'énergétique globale de l'atmosphère fait de celui-ci une composante cruciale des modèles de climat. Nous passerons d'abord en revue les aspects les plus importants de sa représentation dans les modèles de grande échelle. Puis nous donnerons les grandes lignes de la façon dont il répond à des changements climatiques globaux, par exemple sous l'hypothèse d'un réchauffement global associé à l'amplification anthropique de l'effet de serre.

\section{II $\square$ REPRÉSENTATION DU CYCLE DE L'EAU DANS LES MODÈLES DE CLIMAT}

\section{- 2.1. Le transport de l'eau dans l'atmosphère}

Dans les modèles de circulation générale atmosphérique servant à étudier le climat et les changements climatiques, la partie adiabatique des équations de la dynamique atmosphérique est traitée, soit par des méthodes de différences finies, soit par des méthodes pseudo-spectrales en harmoniques sphériques. La deuxième approche n'est guère adaptée au traitement du transport de l'eau (vapeur, eau liquide, cristaux de glace) ni, plus généralement, au transport de quantités positives dont la variance est de l'ordre de la valeur moyenne : les oscillations bien connues de Gibbs produisent des valeurs négatives isolées sans signification physique. Dans la plupart des modèles, $y$ compris les modèles pseudospectraux, le transport de l'eau est donc traité par des méthodes de différences finies. Les schémas de différences utilisés sont bien sûr choisis pour éviter la production d'oscillations parasites et de valeurs négatives, tout en assurant la conservation exacte de la masse d'eau transportée : les schémas centrés sont exclus, les schémas fortement décentrés comme le schéma amont sont acceptables mais trop diffusifs. La préférence va à des méthodes de flux corrigés, comme le schéma de Prather [1] qui a le défaut d'introduire une équation séparée pour la variance, ou, plus simplement, le schéma de van Leer [2]. Une autre approche privilégie les méthodes semi-lagrangiennes.

\section{- 2.2 Les nuages}

La simulation numérique du climat nécessitant des intégrations longues (dix ans, cent ans, quelquefois plus), voire des ensembles d'intégrations longues indépendantes les unes des autres, la résolution des modèles est limitée par le coût du calcul. Les mailles sont de l'ordre de 200 à $400 \mathrm{~km}$, à comparer à une centaine de kilomètres pour les modèles de 


\section{LMD GCM Precipitation (JJA) $\mathrm{mm} /$ day}
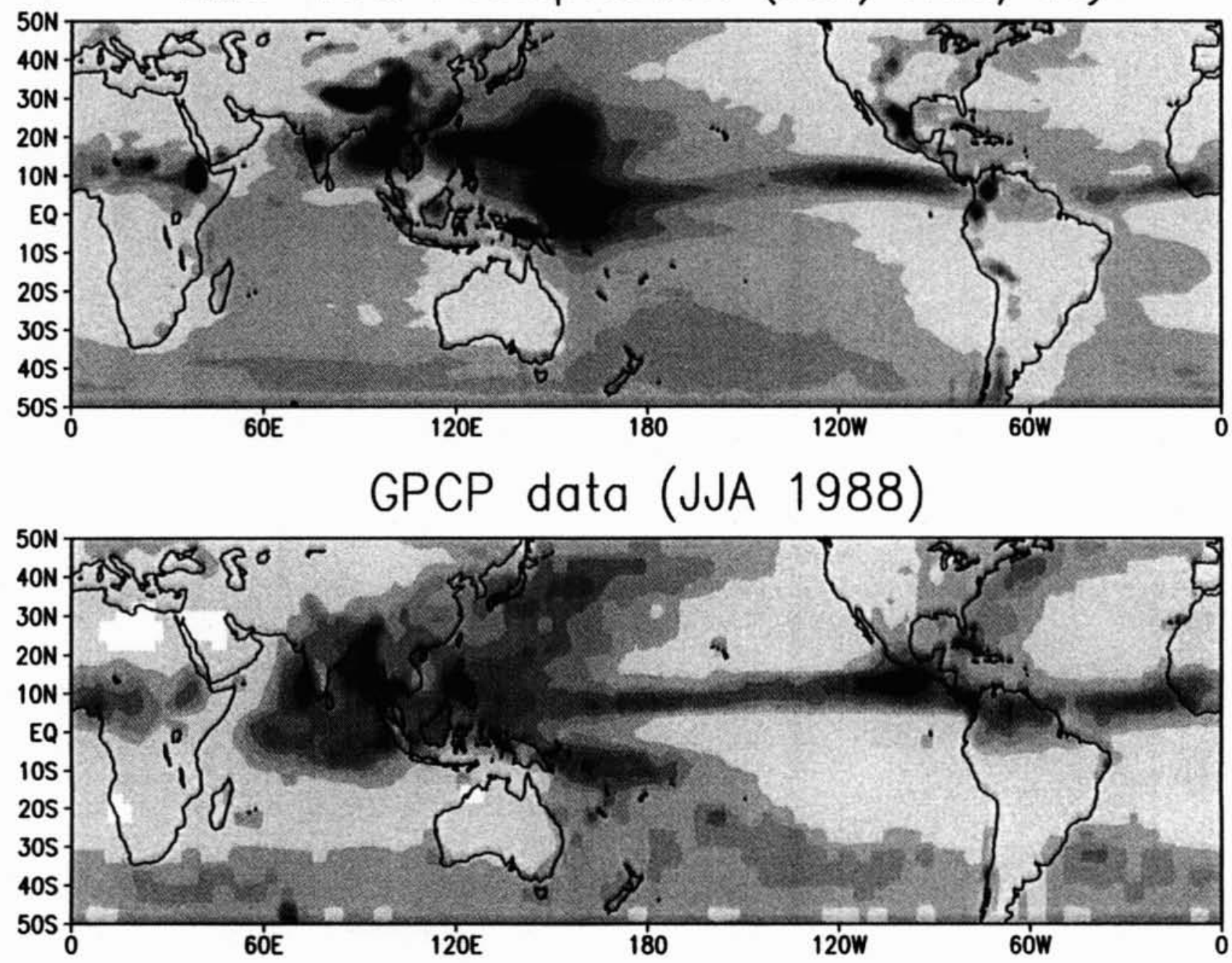

Legates-Willmot Climatology (JJA)

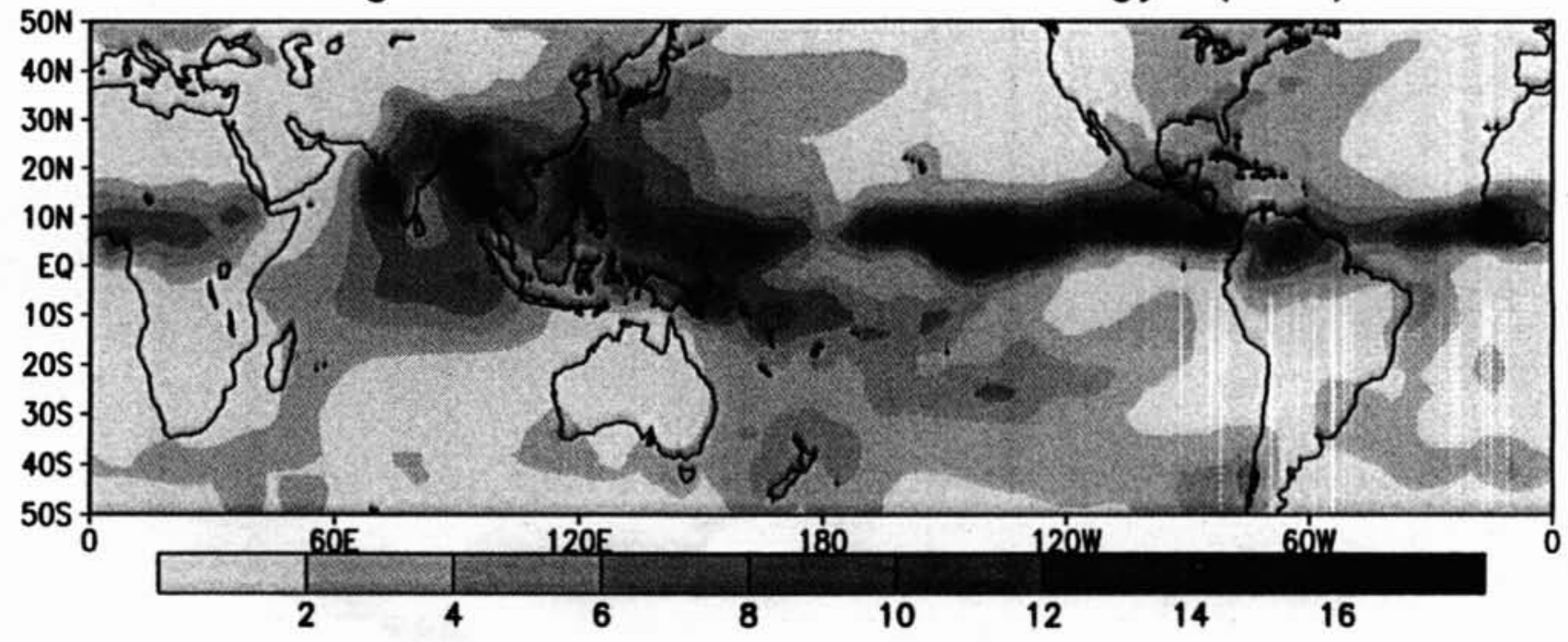

1. Précipitations moyennes simulées pour les mois de juin-juillet-août, par le modèle de circulation générale du LMDCNRS forcé par les températures observées de l'océan, comparées aux observations pour la même période (années 1986 à 1989). 
prévision opérationnelle du temps. À ces échelles, les nuages comme beaucoup d'autres structures physiques, ne peuvent pas être traités individuellement ni modélisés de façon explicite. On doit se contenter de modéliser des distributions de probabilités de couverture nuageuse à l'intérieur de mailles tridimensionnelles, en estimant au mieux les propriétés radiatives associées.

On distingue les nuages stratiformes et les nuages convectifs. Les premiers peuvent être diagnostiqués en fonction des propriétés thermodynamiques locales, à l'intérieur de la maille, la quantité d'eau condensée dépendant de la quantité moyenne de vapeur d'eau dans la maille (prédite par les équations de transport du modèle), de la température et de la pression locales, et de la distribution de probabilité estimée de la vapeur d'eau à l'intérieur de la maille. Les quantités d'eau sous phase liquide ou solide sont des variables pronostiques du modèle, et sont à leur tour transportées. Les nuages convectifs sont modélisés de façon très différente, car ils mettent en jeu des mélanges turbulents verticaux, qui dépendent de l'instabilité de la colonne fluide, c'est-à-dire des gradients verticaux de température et d'humidité. Ces échanges turbulents verticaux ne sont pas pris en compte dans le transport à grande échelle, considéré comme hydrostatique, et doivent donc être modélisés indépendamment, comme la turbulence de couche limite. Il existe de nombreuses méthodes pour modéliser la convection nuageuse, depuis le simple ajustement convectif des profils de température et d'humidité [3], jusqu'aux modèles qui déterminent explicitement la distribution verticale des flux $[4,5]$, et aux modèles conceptuels qui tiennent compte de l'organisation des systèmes convectifs, avec leurs courants ascendants et leurs courants descendants.

Une fois calculée la distribution de probabilité des nuages à l'intérieur d'une maille tridimensionnelle, il reste à définir les propriétés radiatives de l'ensemble. Cela signifie en particulier définir la structure verticale des empilements de nuages (les nuages sont-ils situés au dessus les uns des autres, ou ont-ils des positions aléatoires et décorrélées dans deux couches superposées ? La réponse dépend des situations et la modélisation doit être définie en conséquence). L'autre problème est l'interaction des propriétés radiatives avec la microphysique : l'albedo notamment dépend de la taille des gouttes, qui dépend elle-même de la quantité de noyaux de condensation. Ces problèmes doivent être traités de façon statistique, à l'échelle de mailles horizontales de 200 à $400 \mathrm{~km}$ (l'épaisseur typique d'une maille étant de l'ordre du kilomètre).

La figure 1 illustre la qualité actuelle des modèles de circulation générale atmosphérique. Elle montre les précipitations moyennes simulées pour l'été boréal par le modèle du Laboratoire de Météorologie Dynamique du CNRS.

\section{- 2.3. Les échanges à la surface et l'hydrologie des sols}

La représentation des échanges à la surface est rendue elle aussi délicate par la grande hétérogénéité des propriétés de surface des terres émergées : relief, albedo, types de sol et de végétation ; l'humidité du sol a également une distribution très hétérogène à cause du caractère intermittent des précipitations.

Le calcul des échanges hydriques et énergétiques à la surface des terres émergées demande une prise en compte précise des caractéristiques du couvert végétal. Les processus à prendre en compte sont notamment : les variations de l'albedo en fonction de la saison, l'interception de la pluie par le feuillage, l'évaporation du feuillage mouillé, la profondeur des racines qui lie l'évaporation à la distribution de l'eau dans le sol, les diverses résistances à l'évaporation mises en œuvre par le couvert végétal (des racines aux stomates des feuilles). Le modèle Sechiba [6] est un bon exemple de l'approche actuelle pour la simulation de ces processus de surface. La surface à l'intérieur d'une maille est divisée en huit types (sept types de végétation plus le sol nu), une proportion donnée de la maille étant affectée à chaque type. Les échanges hydriques sont calculés séparément pour chacun de ces types, en accord avec les modèles agrométéorologiques.

Le réalisme de la modélisation de l'hydrologie des sols est évidemment très limité par l'extension des mailles des modèles de climat bien au delà des bassins versants. Un des problèmes est la prise en compte de l'intermittence des précipitations et de l'hétérogénéité qui en résulte pour l'humidité du sol. La non-linéarité très forte des processus en jeu (seuils) rend cette hétérogénéité très importante. Les modèles de sol actuellement utilisés sont multicouches, pour séparer l'assèchement de surface des couches plus profondes, en liaison avec la prise en compte de systèmes racinaires dans l'évapotranspiration. Enfin, un point important est le calcul du drainage et du ruissellement, modélisé bien sûr à l'échelle des mailles en fonction de la distribution des pentes et des types de sol dans la maille.

\subsection{Les échanges à travers la couche limite atmosphérique}

La couche limite atmosphérique constitue l'interface dynamique entre les processus de surface d'une part, la convection et la circulation de grande échelle d'autre part. Comme pour la convection, il s'agit de représenter des mouvements tridimensionnels éventuellement turbulents, non hydrostatiques, qui donc échappent au formalisme de la circulation de grande échelle et doivent être modélisés séparément. Les modèles les plus simples sont de simples modèles diffusifs, dans lesquels le cœefficient de diffusion verticale dépend du nombre de Richardson (qui mesure l'importance relative de la stabilité thermique par rapport au cisaillement vertical de la vitesse horizontale). D'autres modèles concentrent l'effort sur le calcul de la hauteur de la couche limite, qu'ils considèrent a priori comme bien mélangée ; l'inconvénient de cette approche est que la hauteur de la couche limite n'est pas toujours bien définie, notamment au cours du cycle diurne quand la couche limite se dilue et se reforme au dessous. La méthode la plus élaborée a recours à une équation pronostique pour l'énergie turbulente, mais la modélisation suivant cette approche n'est pas toujours bien conditionnée.

\section{III — IMPACTS PRÉVISIBLES DES CHANGEMENTS CLIMATIQUES}

\section{- 3.1. Des changements climatiques naturels aux changements anthropiques}

Il y a eu, dans le passé relativement récent, d'importants changements climatiques qui ont affecté, de façon quelquefois dramatique, les conditions de vie des habitants de la pla- 
nète. L'alternance des périodes glaciaires et interglaciaires sous l'effet des variations d'ensoleillement dues aux perturbations du mouvement orbital de la Terre au cours des deux derniers millions d'années, ou, encore plus près de nous, le refroidissement du climat entre le quinzième siècle et le dixneuvième siècle, appelé de façon imagée «petit âge glaciaire», et probablement lié, au moins dans sa phase la plus extrême, à une baisse sensible de l'activité solaire, sont les exemples les plus connus de ces changements naturels du climat. La dernière période glaciaire, initiée il y a 115000 ans, a connu son maximum il y a 20000 ans avec des calottes glaciaires de l'ordre de deux à trois kilomètres d'épaisseur sur le Canada et le nord de l'Europe, jusqu'à l'Angleterre et la Hollande. La température moyenne à la surface était de l'ordre de cinq degrés au dessous de l'actuelle, les pôles étant plus froids qu'aujourd'hui d'environ dix degrés. Au dix-septième siècle, la période la plus aiguë du petit âge glaciaire, les températures moyennes sur l'Europe de l'ouest étaient d'un à deux degrés plus froides qu'aujourd'hui ; cette période froide a été abondamment illustrée par les scènes de patinage sur les lacs et les canaux gelés, si fréquentes chez les peintres flamands de l'époque. La fin du petit âge glaciaire au sens large est elle aussi illustrée par le recul des glaciers alpins du dix-neuvième au vingtième siècle.

Quant à la période actuelle, elle est surtout marquée par une explosion démographique à l'échelle de la planète, accompagnée d'un développement extrêmement rapide de l'industrie, de l'agriculture et des transports, avec des incidences également planétaires. Cette évolution a des effets indirects, dont les plus notables sont l'injection dans l'atmosphère de gaz à effet de serre comme $\mathrm{CO}_{2}, \mathrm{CH}_{4}, \mathrm{~N}_{2} \mathrm{O}, \mathrm{O}_{3}$, les chlorofluorocarbures ou leurs substituts. Les injections actuelles de $\mathrm{CO}_{2}$ dans l'atmosphère par les transports, l'industrie, le chauffage et la combustion de biomasse, correspondent à sept milliards de tonnes de carbone par an ; la moitié est absorbée par l'océan et la biosphère continentale, l'autre moitié (trois milliards et demi de tonnes) s'accumule et produit la croissance actuellement observée. Depuis un siècle et demi, c'est-à-dire depuis le début de l'ère industrielle, la teneur de l'atmosphère en $\mathrm{CO}_{2}$ a augmenté d'un tiers (de 280 à 360 parties par million en volume), la teneur en $\mathrm{CH}_{4}$ a doublé (de 0,8 à 1,6 parties par million en volume) ; quant aux autres gaz à effet de serre, ils étaient pratiquement absents dans l'atmosphère pré-industrielle. La perturbation du bilan radiatif induite par ces augmentations est relativement faible, mais systématique : de l'ordre de $1,5 \mathrm{~W} \mathrm{~m}^{-2}$. Ce chiffre paraît petit comparé aux $240 \mathrm{~W} \mathrm{~m}^{-2}$ du flux net infrarouge sortant, mais est la source d'une rupture d'équilibre du système qui conduit à son réchauffement. Les scénarios actuels pour les émissions futures de $\mathrm{CO}_{2}$ dans l'atmosphère donnent, sous des hypothèses vraisemblables, un doublement du $\mathrm{CO}_{2}$ atmosphérique vers le milieu du siècle prochain. Ce doublement correspondra à une perturbation radiative (flux additionnel dirigé vers le bas à la tropopause) de l'ordre de $4 \mathrm{~W} \mathrm{~m}^{-2}$. Les estimations actuelles du réchauffement climatique moyen global à la surface, associé à une telle perturbation, sont comprises entre 1,5 et $4,5^{\circ} \mathrm{C}$. L'incertitude provient principalement de la façon dont sont modélisés les nuages et leurs propriétés radiatives.

L'activité des hommes ne se traduit pas seulement par l'émission de gaz à effet de serre. Les diverses formes de combustion relâchent aussi dans l'atmosphère des aérosols de diverses natures, dont un des effets est de faire écran au rayonnement solaire et de refroidir ainsi les basses couches.
Cet effet est donc contraire au précédent. L'étude des aérosols et de leurs effets radiatifs et climatiques est assez complexe et n'a été pour l'instant menée (et encore, de façon très incomplète) que pour les aérosols soufrés [7]. On distingue deux effets : l'effet direct, qui est simplement la rétrodiffusion accompagnée d'extinction partielle du flux solaire par les aérosols eux-mêmes, et l'effet indirect, dû au rôle des aérosols soufrés comme noyaux de condensation. La présence d'aérosols en très grandes quantités augmente le nombre de gouttelettes d'eau pour un volume donné d'eau condensée ; quand le nombre de gouttes augmente, la taille des gouttes diminue et la surface de l'ensemble des gouttes augmente comme la racine troisième du nombre de gouttes. La présence de grandes quantités de noyaux de condensation rend donc les nuages plus réfléchissants. Le double effet des aérosols soufrés va donc dans le sens d'un refroidissement des basses couches et s'oppose ainsi à l'effet de serre additionnel anthropique [8]. Les simulations comparées des deux effets indiquent que, en moyenne globale, le refroidissement dû aux émissions d'aérosols soufrés depuis le début de l'ère industrielle a à peu près compensé le réchauffement dû aux émissions de gaz à effet de serre. La grande différence entre les deux effets est que le temps de résidence dans l'atmosphère des aérosols soufrés est de l'ordre de la semaine (ce sont des noyaux de condensation potentiels et ils sont donc rapidement lessivés par les pluies), contre sept ans environ pour le $\mathrm{CO}_{2}$. Il s'ensuit que, tandis que le $\mathrm{CO}_{2}$ est à peu près uniformément réparti sur la planète, les panaches d'aérosols soufrés restent dans le voisinage des sources : le nord-est de l'Amérique du Nord, l'Europe centrale et l'Asie du sud-est. L'impact sur le rayonnement solaire est bien sûr concentré sur les panaches. L'impact climatique est plus diffus à cause des mouvements de I'atmosphère qui peuvent transporter l'énergie sur de grandes distances. Mais le contraste entre l'hémisphère nord, très pollué par les aérosols, et l'hémisphère sud, relativement pur, reste bien marqué au niveau de la réponse climatique. L'hémisphère nord se serait plutôt refroidi sous l'effet combiné des aérosols et des gaz à effet de serre, 1'hémisphère sud se serait plutôt réchauffé [9].

\subsection{Incertitudes quantitatives sur l'amplitude de la réponse climatique}

On a vu plus haut que la source majeure d'incertitude sur l'amplitude de la réponse du climat à une perturbation radiative est liée à la modélisation des nuages. On a vu également que les nuages jouaient un double rôle : un rôle refroidissant par leur effet d'albedo (rétrodiffusion de l'énergie solaire par les hydrométéores), un rôle réchauffant par leur effet de serre (absorption du rayonnement tellurique dans l'infrarouge). Plus les nuages montent haut, plus leur sommet est froid ; d'après la loi de Planck $\left(R=\sigma T^{4}\right)$ les nuages hauts émettent donc faiblement dans l'infrarouge, c'est-à-dire ont un effet de serre fort. Les nuages bas, au contraire, ont un effet de serre faible, car leur température d'émission n'est pas très différente de celle du sol. D'autre part, les nuages bas, plus chargés en eau, réfléchissent plus fortement le rayonnement solaire. Pour toutes ces raisons, les nuages hauts sont des agents de réchauffement, les nuages bas des agents de refroidissement. Lors de changements climatiques, la structure verticale des nuages est modifiée et leurs propriétés radiatives peuvent donc être fortement perturbées : c'est ce que l'on appelle les effets de rétroaction liés aux nuages. Ces rétroactions peuvent être très différentes suivant la manière dont ils sont modélisés. 


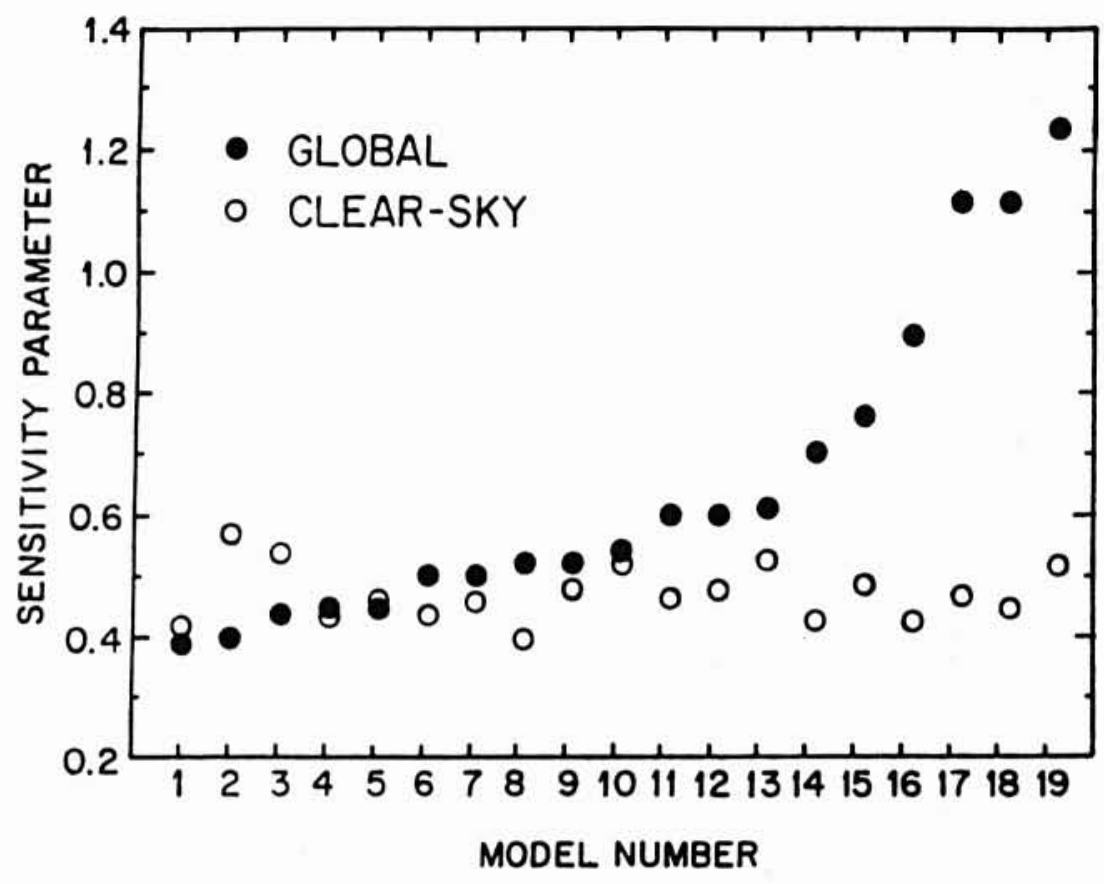

2. Dispersion de la sensibilité climatique $S$ pour 19 modèles, en $\mathrm{W}$ $\mathbf{m}^{-2}$, en l'absence de nuages (cercles blancs) et en présence de nuages (cercles noirs). D'après [10].

La figure 2 illustre cette sensibilité à la modélisation des nuages, d'après Cess et al. |10|. La réponse moyenne du climat à une perturbation radiative $d R$ est mesurée par la quantité $S=d T_{S} / d R$, où $d T_{S}$ est la variation de la température moyenne à la surface induite par $d R ; S$ (appelé ici paramètre de sensibilité climatique) intègre donc l'ensemble de tous les mécanismes de rétroaction qui interviennent dans la dynamique du changement climatique, parmi lesquels bien sûr ceux liés à la modélisation des nuages. La figure 2 montre les valeurs de $S$ calculées par 19 modèles, dans deux cas de figure :

(i) en tenant compte des rétroactions dues aux nuages

(ii) en supposant le ciel clair.

On voit que les modèles s'accordent entre eux sur la réponse en ciel clair, et diffèrent d'un facteur trois sur la réponse «globale», c'est-à-dire tenant compte de l'effet des nuages.
- 3.3. Caractéristiques générales de la réponse du cycle de l'eau à un réchauffement global du climat

Le caractère cumulatif de l'augmentation des gaz à effet de serre, lié à la longue durée de vie de ces gaz dans l'atmosphère, fait que l'effet de réchauffement l'emportera progressivement sur le refroidissement associé aux émissions d'aérosols troposphériques qui l'a plus ou moins contrebalancé jusqu'à présent. On se placera donc ici sous I'hypothèse d'une perturbation radiative du type réchauffement, et on donnera une esquisse simplifiée de la réponse du cycle de l'eau. Les résultats seront présentés sous forme quantitative, mais il ne faudra pas oublier que l'amplitude des réponses dépend du modèle utilisé, en vertu des incertitudes énoncées plus haut.

On se placera dans le cadre d'une perturbation radiative de I $\mathrm{W} \mathrm{m}^{-2}$, dirigée vers le bas, à l'altitude de la tropopause ; il

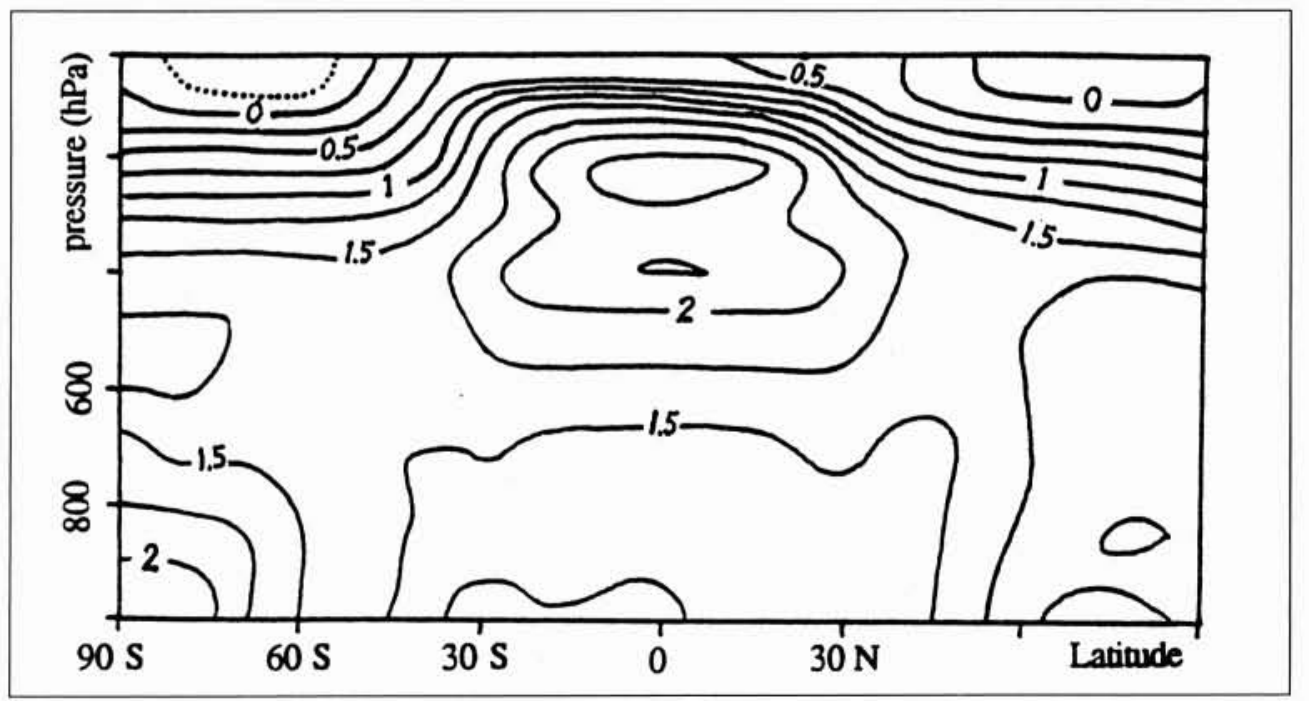

3. Structure méridienne d'un réchauffement climatique global, en $\mathbf{K} /\left(\mathbf{W ~ m}^{-2}\right)$, en moyenne annuelle. D'après [11]. 
s'agit donc a priori d'un réchauffement. Cette perturbation peut être attribuée, soit à une accumulation de gaz à effet de serre (c'est alors une diminution du flux infrarouge émergeant), soit à une augmentation d'environ $0,4 \%$ de l'irradiance solaire (du même ordre que celle qui aurait pu être observée lors du minimum de Maunder). Des simulations indiquent que ces deux types de perturbation, a priori assez différents, induisent des changements climatiques analogues à la surface. Les résultats qui suivent sont adaptés de Nesme-Ribes et al. [11], qui étudiait l'impact d'une variation de l'irradiance solaire ; mais ils présentent une certaine généralité.

La structure méridienne du réchauffement $d T / d R$, en $\mathrm{K}$ par (W $\mathrm{m}^{-2}$ ), est illustrée sur la figure 3. Il s'agit d'une moyenne annuelle. On notera que le maximum de réchauffement dans les basses couches se situe aux hautes latitudes, à cause de l'interaction du réchauffement avec la fonte de la neige : sur un sol enneigé, l'absorption d'un excédent d'énergie provoque une augmentation de la fonte, qui entraîne une diminution de l'albedo de la surface et par conséquent une absorption d'énergie encore plus grande. Ce type de mécanisme est appelé rétroaction positive, il induit une amplification de la réponse, aux hautes latitudes où il $\mathrm{y}$ a de la neige pendant une partie de l'année. L'autre caractéristique de la figure est la présence d'un maximum de réchauffement dans la haute troposphère tropicale. Cette structure résulte de l'amplification de la convection tropicale humide : tout réchauffement, même léger, dans les tropiques induit une très sensible humidification des basses couches de l'atmo- sphère, à cause de la dépendance exponentielle en température du rapport de mélange d'humidité saturante, donnée par la loi de Clausius-Clapeyron. Cette forte humidification conduit à une instabilité plus forte et à une convection plus active, entraînant des précipitations plus intenses et à un dégagement de chaleur latente plus puissant, qui réchauffe les hautes couches.

Les cartes du réchauffement en surface et du contenu en eau de l'atmosphère (figure 4) montrent bien des comportements opposés, avec le maximum de réchauffement aux hautes latitudes pour l'une, et un maximum d'humidification dans les tropiques pour l'autre. L'amplification des phénomènes convectifs tropicaux est a priori cohérent avec une intensification des phénomènes de mousson. Cela semble vérifié en ce qui concerne la mousson indienne d'été, qui paraît effectivement plus active dans la plupart des simulations d'un réchauffement climatique global.

Il est bien sûr particulièrement important d'étudier l'impact du réchauffement sur les ressources en eau du sol. Les variations d'humidité du sol associées au réchauffement climatique sont illustrées par la figure 5 . On notera l'assèchement du sol pendant l'été, et même pendant l'hiver dans les subtropiques et notamment, sur le pourtour de la Méditerranée, ce qui peut impliquer à terme une désertification de ces régions. Par contre, on observe une humidification importante du sol pendant l'hiver, dans tout le centre et l'est de l'Europe. Les changements hivernaux de l'humidité des sols sont liés à des modifications significatives de la circula-

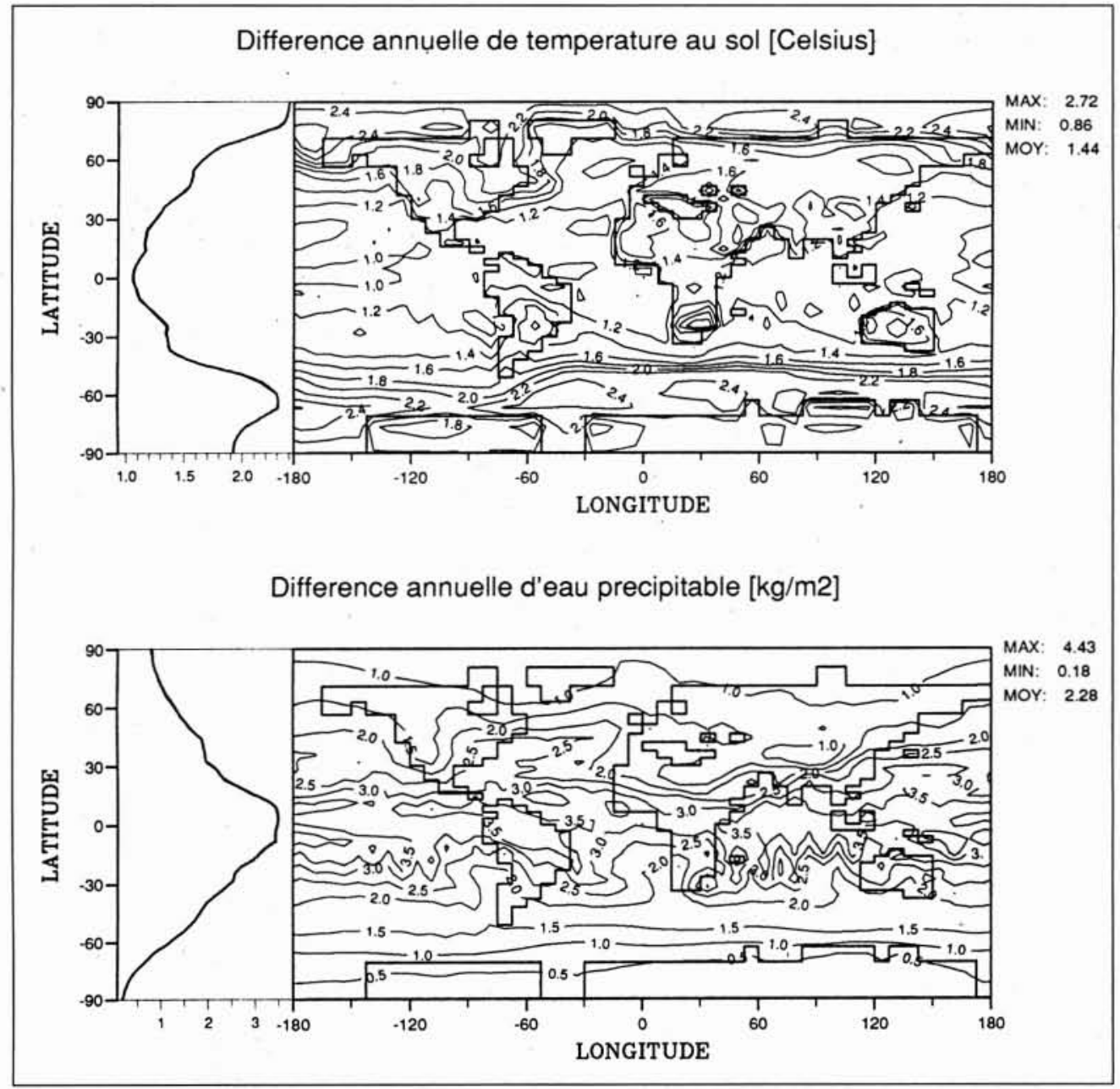

4. Structure du réchauffement à la surface, en $\mathbf{K} /\left(W^{-2}\right)$, en haut, et de l'humidification de l'atmosphère (en mm d'eau par $W^{-2}$ ), en bas, tous deux en moyenne annuelle. D'après [11]. 


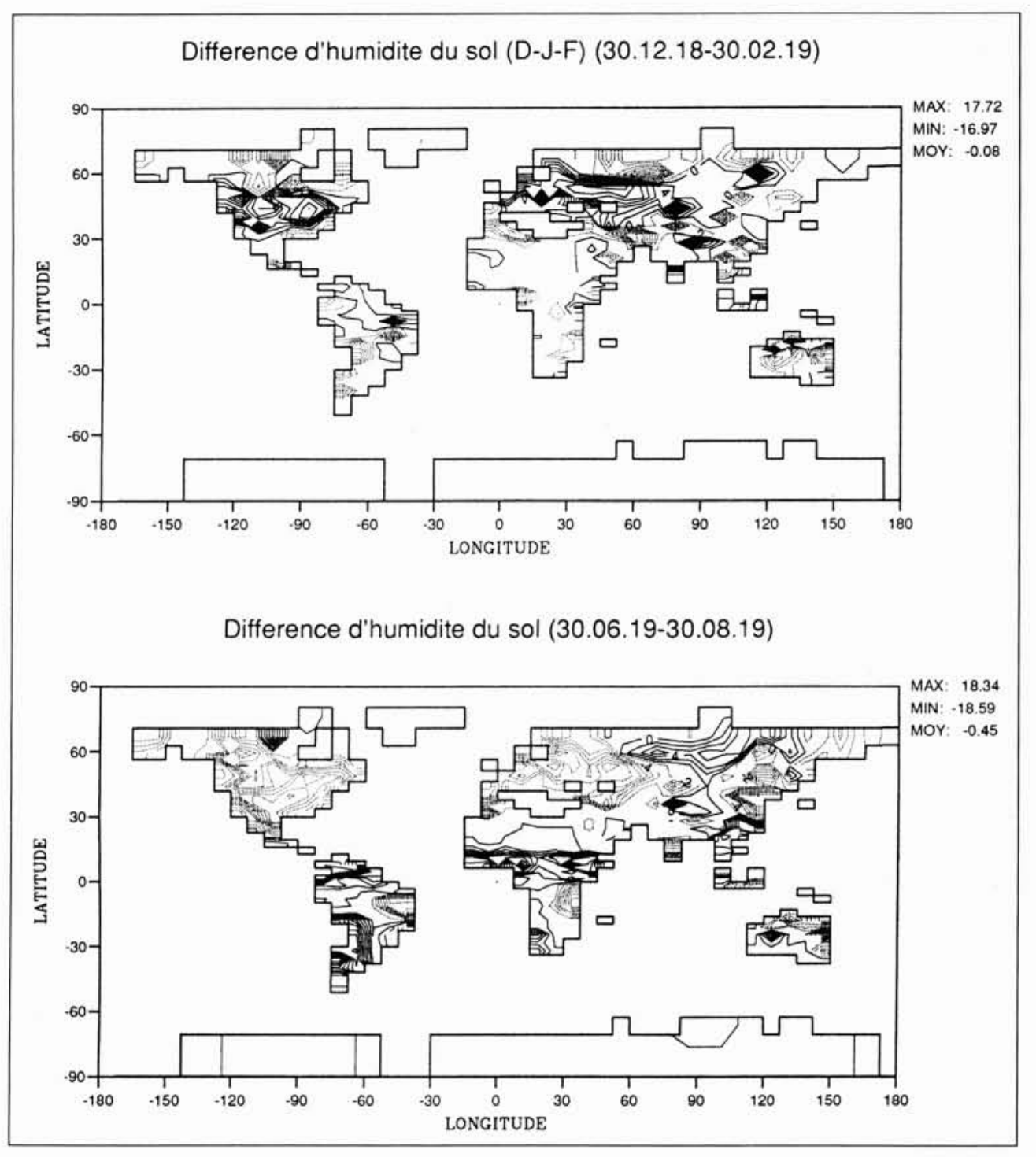

5. Variation de l'humidité du sol, en $\mathrm{mm}$ d'eau par $\mathbf{W} \mathbf{m}^{-2}$. De haut en bas : hiver boréal, été boréal. D'après [11].

tion générale de l'atmosphère. L'assèchement des confins de la Méditerranée est cohérent avec une extension vers le nord des subsidences de la circulation de Hadley, elle-même causée par le réchauffement des hautes couches de l'atmosphère tropicale mentionné plus haut. L'humidification de l'Europe centrale est cohérent avec un transport plus intense de vapeur d'eau vers le pôle, répondant à l'humidification des basses couches de l'atmosphère tropicale et à l'intensification qui en résulte pour le gradient d'humidité entre le pôle et l'équateur.

\section{IV $\square$ CONCLUSION}

La représentation des divers processus intervenant sur le cycle de l'eau dans les modèles de circulation générale atmosphérique a été très fortement améliorée au cours de ces dernnières années. La couverture nuageuse et ses propriétés radiatives sont aujourd hui explicitement calculées, avec une ébauche de traitement des propriétés microphysiques. Les processus de surface sont également traités de façon de plus en plus réaliste, avec la prise en compte des divers types de végétation, et de l'hydrologie des sols. La disparité d'échelles entre la plupart de ces processus et les mailles des modèles, si elle complique le problème, n'est pas un réel obstacle à des modélisations physiques et relativement réalistes de tous ces processus.

Ces avancées progressives font aujourd'hui des modèles de climat à grande échelle des outils adaptés au traitement du problème majeur qu'est le changement climatique, même si demeurent des incertitudes d'ordre quantitatif dues à la complexité des rétroactions qui jouent sur l'amplitude des phénomènes. Une des certitudes dans le domaine de la dynamique du climat, est que le cycle de l'eau joue un grand rôle dans les variations du climat, et qu'il est extrêmement sensible aux perturbations radiatives globales, telles que l'augmentation anthropique de l'effet de serre. On a tenté de décrire ici les grandes lignes de cette sensibilité. 


\section{Bibliographie}

[1] Prather, M., 1988 : Numerical advection by conservation of second order moments. J. Geophys. Res., 91, 6671-6681.

[2] van Leer, B., 1977 : Towards the ultimate conservative difference scheme, IV : A new approach to numerical convection. J. Comput. Phys. 23, 276-299.

[3] Manabe, S. and R.F. Strickler, 1964 : Thermal equilibrium of the atmosphere with the convective adjustment. J. Atmos. Sci. 21, 361-385.

[4] Arakawa, A. and W.H. Schubert, 1974 : Interaction of cumulus cloud ensemble with the large-scale environment. J. Atmos. Sci. 31, 674-701.

[5] Large-scale models. Mon. Wea. Rev. 117, 1779-1800.

[6] Ducoudré, N., K. Laval and A. Perrier, 1991 : A new set of parameterizations of the hydrologic exchanges at the land-atmosphere interface within the LMD atmospheric general circulation model. J. Climate 6, 248273.
[7] Charlson, R.J., J. Langer, H. Rodhe, C.B. Leovy and S.G. Warren, 1991 : Perturbation of the Northern Hemisphere radiative balance by backscattering from anthropogenic sulfate aerosols. Tellus 43 AB, 152-163.

[8] Charlson, R.J., S.E. Schwartz, J.M. Hales, R.D. Cess, J.A. Coakley, Jr., J.E. Hansen and D.J. Hofmann, 1992 : Climate forcing by anthropogenic aerosols. Science 255, 422-430.

[9] Boucher, O., H. Le Treut and M.B. Baker 1995 : Precipitation and radiation modelling in a GCM : Introduction to cloud microphysics. J. Geophys. Res. 100, 16395-16414.

[10] Cess, R.D. et al., 1990 : Intercomparison and interpretation of climate feedback processes in nineteen atmospheric general circulation models ; J. Geophys. Res. 95, 16601-16615.

[11] Nesme-Ribes, E., E. Ferreira, R. Sadourny, H. Le Treut and Z.X. Li, 1993 : Solar dynamics and its impact on solar irradiance and the terrestrial climate. J. Geophys. Res. 98 A, 18923-18935. 